\title{
On the number of countable models of stable theories
}

\author{
by \\ Predrag Tanović (Beograd)
}

\begin{abstract}
We prove:
THEOREM. If $T$ is a countable, complete, stable, first-order theory having an infinite set of constants with different interpretations, then $I\left(T, \aleph_{0}\right) \geq \aleph_{0}$.

Let $T$ be a countable, complete, first-order theory having infinite models, and let $I\left(T, \aleph_{0}\right)$ denote the number of isomorphism classes of its countable models. There are several known examples where $I\left(T, \aleph_{0}\right)$ is finite and greater than 1 . They are obtained by first constructing an $\aleph_{0}$-categorical theory and then adding an infinite set of constants. In all of them the underlying theory interprets a partial order with infinite chains; in particular they are unstable (an infinite subset of a model of $T$ is linearly ordered by a formula). It has been conjectured that it is not possible to find such an example with $T$ stable:
\end{abstract}

(C) If $T$ is stable and not $\aleph_{0}$-categorical then $I\left(T, \aleph_{0}\right) \geq \aleph_{0}$.

There are a few results partially confirming conjecture (C): Lachlan [4] proved it for superstable theories, Pillay [6] for weakly normal theories, Hrushovski [2] for theories which admit finite coding, Herwig and others [3] for theories with no dense forking chains, Tsuboi [7] for unions of $\aleph_{0}$-categorical theories, and Tsuboi [8] for unions of pseudo-superstable theories. In this paper we confirm the conjecture for theories having an infinite set of constants with different interpretations.

We shall assume that the reader is familiar with some basic stability theory, as can be found in [1] or [5]. Throughout the paper let $T$ be a fixed, stable and small (i.e $|S(\emptyset)| \leq \aleph_{0}$ ) theory, and we operate in its monster model $\mathcal{M}$; all the sets are countable subsets of $\mathcal{M}$, all the models are elementary submodels of $\mathcal{M}$ and $=\phi(\bar{a}, \bar{b})$ means $\mathcal{M}=\phi(\bar{a}, \bar{b})$. The smallness

2000 Mathematics Subject Classification: Primary 03C45; Secondary 03C15.

Supported by Ministry of Science and Technology of Serbia. 
of $T$ implies existence of atomic (hence prime) models over finite sets and the Cantor-Bendixson rank, denoted by $\mathrm{CB}(-)$, has ordinal value on $S(\emptyset)$.

The independence of $A$ and $B$ over $C$ is denoted by $A \downarrow B(C)$, the dependence by $A \notin B(C)$; two types $p \in S(A)$ and $q \in S(B)$ are almost orthogonal, $p \perp^{\mathrm{a}} q$, if whenever $\bar{a}$ and $\bar{b}$ realize nonforking extensions of $p$ and $q$ to $A B$, then $\bar{a} \downarrow \bar{b}(A B)$. We use Hrushovski's quantifier: if $p \in S(A)$ is stationary and $\phi(\bar{x}, \bar{y})$ is over $A$ then $\left(d_{p} \bar{x}\right) \phi(\bar{x}, \bar{y})$ is read "for the generic $\bar{x}$ satisfying $p$ ", meaning: $=\left(d_{p} \bar{x}\right) \phi(\bar{x}, \bar{b})$ iff for some (all) $\bar{a} \models p, \bar{a} \downarrow \bar{b}(A)$ implies $\phi(\bar{a}, \bar{b})$. Finally, $\bar{a}$ is definable if there exists a formula $\phi(\bar{x})$ without parameters such that $\bar{a}$ is the only tuple of elements of $\mathcal{M}$ satisfying $\phi(\bar{x})$.

Definition. A type is strongly nonisolated if it is almost orthogonal to all isolated types over the same or a larger domain.

We list some basic properties of strongly nonisolated types that will be used further in the text without specific mentioning:

(1) A nonforking extension of a strongly nonisolated type is strongly nonisolated.

(2) If $\operatorname{tp}(\bar{a})$ is stationary and strongly nonisolated and $\operatorname{tp}(\bar{b})$ is isolated then $\operatorname{tp}(\bar{b} / \bar{a})$ is isolated. To see this, note that $\bar{a}$ and $\bar{b}$ must be independent so, by stationarity, there is only one possibility for $\operatorname{tp}(\bar{a} / \bar{b})$ and hence for $\operatorname{tp}(\bar{a} \bar{b})$ as well. Therefore $\operatorname{tp}_{\bar{x}}(\bar{a}) \cup \operatorname{tp}_{\bar{y}}(\bar{b}) \vdash \operatorname{tp}_{\bar{x} \bar{y}}(\bar{a} \bar{b})$ and $\operatorname{tp}(\bar{b} / \bar{a})$ is isolated.

(3) A power of a stationary, strongly nonisolated type is also strongly nonisolated. To see this, suppose that $\bar{a}_{1}$ and $\bar{a}_{2}$ are two independent realizations of such a type and let $\operatorname{tp}(\bar{b})$ be isolated. Then $\operatorname{tp}\left(\bar{a}_{1} / \bar{a}_{2}\right)$ is strongly nonisolated by (1), and $\operatorname{tp}\left(\bar{b} / \bar{a}_{2}\right)$ is isolated by (2). Thus $\bar{a}_{1} \downarrow \bar{b}\left(\bar{a}_{2}\right)$ and since $\bar{b} \downarrow \bar{a}_{2}$ we must have $\bar{a}_{1} \bar{a}_{2} \downarrow \bar{b}$. Therefore $\operatorname{tp}\left(\bar{a}_{1}\right)^{2}$ is strongly nonisolated and the general case follows by induction.

Lemma 1. Suppose that $p \in S_{1}(\emptyset)$ is an accumulation point of types of definable elements. Then $p$ is stationary and strongly nonisolated.

Proof. Suppose that $E$ is a definable equivalence relation with finitely many classes and choose a sequence of types of definable elements converging to $p$. Then almost all elements chosen are in the same $E$-class, since the sequence is convergent. All the realizations of $p$ have to be in the class. Thus $p$ is stationary and it remains to show that $p$ is strongly nonisolated.

Suppose, on the contrary, that $q \in S_{m}(\emptyset)$ is isolated, by $\varphi(\bar{y})$ say, $a=p$, $\bar{b}=q$ and $a$ and $\bar{b}$ are dependent. Let $\psi(x, \bar{y})$ be such that $\models \psi(a, \bar{b})$ and $\psi(x, \bar{b})$ forks over $\emptyset$. Consider the formula

$$
(\exists \bar{y})(\varphi(\bar{y}) \wedge \psi(x, \bar{y})) .
$$

It is satisfied by $a$, so it belongs to $p$. Hence, there exists a definable element $c$ which satisfies our formula. Pick $\bar{b}^{\prime}$ so that $\models \varphi\left(\bar{b}^{\prime}\right) \wedge \psi\left(c, \bar{b}^{\prime}\right)$. Since $\operatorname{tp}(\bar{b})=$ 
$\operatorname{tp}\left(\bar{b}^{\prime}\right), \psi\left(x, \bar{b}^{\prime}\right)$ forks over $\emptyset$, so $c$ and $\bar{b}^{\prime}$ are dependent, contradicting the definability of $c$.

Therefore, $p$ is almost orthogonal to all isolated types from $S(\emptyset)$. Since any nonforking extension of $p$ is an accumulation point of types of definable elements, $p$ is strongly nonisolated.

In order to prove the Theorem we shall prove the following, more general result, from which the Theorem follows according to Lemma 1:

THEOREM 1. Suppose that there exists a stationary, strongly nonisolated type over $\emptyset$. Then $I\left(T, \aleph_{0}\right) \geq \aleph_{0}$.

In the proof of Theorem 1 the key role will be played by $p$-minimal types; the notion is introduced below. In the absence of regular types some of their good properties, proved in Lemmas 2 and 3, will enable us to construct infinitely many nonisomorphic models.

Definition. Let $p \in S(\emptyset), q \in S_{n}(A)$.

(a) $q$ is $p$-minimal if there exists a formula $\varphi(\bar{x})$ such that $q$ is the only type in $S_{n}(A)$ which contains $\varphi(\bar{x})$ and is not almost orthogonal to $p$.

(b) The sequence $\left(\bar{a}_{1}, \bar{a}_{2}, \ldots, \bar{a}_{n}\right)$ is p-minimal if $\operatorname{tp}\left(\bar{a}_{1}\right), \operatorname{tp}\left(\bar{a}_{2} / \bar{a}_{1}\right), \ldots$ $\ldots, \operatorname{tp}\left(\bar{a}_{n} / \bar{a}_{1} \bar{a}_{2} \ldots \bar{a}_{n-1}\right)$ are all $p$-minimal.

Note that for any $p$, since $T$ is small, $p$-minimal types (over $\operatorname{dom}(p)$ ) exist: take a type of minimal CB-rank which is not almost orthogonal to $p$.

We shall be interested in $p$-minimal types when $p$ is strongly nonisolated, in which case, since $p$ is almost orthogonal to all isolated types, they must be nonisolated.

Recall that $\bar{b}$ is semi-isolated over $\bar{a}$ if there exists a formula $\phi(\bar{x}, \bar{a}) \in$ $\operatorname{tp}(\bar{b} / \bar{a})$ such that $\phi(\bar{x}, \bar{a}) \vdash \operatorname{tp}(\bar{b})$.

Lemma 2. Suppose that $p, q \in S(\emptyset), p$ is strongly nonisolated, $q$ is $p$ minimal, $\bar{a}=p, \bar{b}=q$ and $\bar{a}$ and $\bar{b}$ are dependent. Then $\bar{b}$ is semi-isolated over $\bar{a}$.

Proof. Suppose $\psi(\bar{y})$ witnesses $p$-minimality of $q$. Let $\varphi(\bar{x}, \bar{a})$ be satisfied by $\bar{b}$ and forking over $\emptyset$ and suppose $\models \varphi\left(\bar{b}^{\prime}, \bar{a}\right) \wedge \psi\left(\bar{b}^{\prime}\right)$. Now $\models \varphi\left(\bar{b}^{\prime}, \bar{a}\right)$ implies that $\bar{b}^{\prime}$ forks with $\bar{a}$ and $\models \psi\left(\bar{b}^{\prime}\right)$ implies that $\bar{b}^{\prime} \models q$ since $q$ is the only type containing $\psi(\bar{x})$ which is not almost orthogonal to $p$. Therefore, $\varphi(\bar{x}, \bar{a}) \wedge \psi(\bar{x}) \vdash q(\bar{x})$ and $\bar{b}$ is semi-isolated over $\bar{a}$ by the latter formula.

It follows from the lemma that whenever $q$ is $p$-minimal and $\bar{a} \models p$ then there is an isolated extension of $q$ in $S(\bar{a})$; take $\bar{b}=q$ which forks with $\bar{a}$ and a formula $\phi(\bar{x}, \bar{a}) \in \operatorname{tp}(\bar{b} / \bar{a})$ implying $q(\bar{x})$, then choose an isolated type in $S(\bar{a})$ containing $\phi(\bar{x}, \bar{a})$. 
Lemma 3. Suppose $p, q \in S(\emptyset), p$ is stationary and strongly nonisolated, $q$ is $p$-minimal, $\bar{b}=q$ and $\bar{a}_{1}$ and $\bar{a}_{2}$ are two independent realizations of $p$. Then $\bar{b}$ is independent of at least one of $\bar{a}_{1}$ and $\bar{a}_{2}$.

Proof. Suppose, on the contrary, that $\bar{b}$ forks with both $\bar{a}_{1}$ and $\bar{a}_{2}$ and choose formulas $\phi_{1}\left(\bar{y}, \bar{a}_{1}\right)$ and $\phi_{2}\left(\bar{y}, \bar{a}_{2}\right)$ both satisfied by $\bar{b}$, both forking over $\emptyset$ and, using Lemma 2, both implying $q(\bar{x})$. Let $\varphi(\bar{x})$ be $\left(d_{p} \bar{z}\right)(\exists \bar{y})\left(\phi_{1}(\bar{y}, \bar{x}) \wedge\right.$ $\left.\phi_{2}(\bar{y}, \bar{z})\right)$. Clearly, $\varphi(\bar{x}) \in p$.

Claim 1. Any type from $S(\emptyset)$ containing $\varphi(\bar{x})$ is consistent with $\phi_{1}(\bar{b}, \bar{x})$.

Proof. Suppose $\models \varphi\left(\bar{a}_{1}^{\prime}\right)$. Choose $\bar{a}_{2}^{\prime} \models p$ independent of $\bar{a}_{1}^{\prime}$ and choose $\bar{b}^{\prime}$ satisfying $=\phi_{1}\left(\bar{b}^{\prime}, \bar{a}_{1}^{\prime}\right) \wedge \phi_{2}\left(\bar{b}^{\prime}, \bar{a}_{2}^{\prime}\right)$. Now $\phi_{2}\left(\bar{b}^{\prime}, \bar{a}_{2}^{\prime}\right)$ ensures that $\bar{b}^{\prime}=q$ hence if $\operatorname{tp}\left(\bar{b}^{\prime} \bar{a}_{1}^{\prime}\right)=\operatorname{tp}\left(\bar{b} \bar{a}_{1}^{\prime \prime}\right)$ we have $\operatorname{tp}\left(\bar{a}_{1}^{\prime \prime}\right)=\operatorname{tp}\left(\bar{a}_{1}^{\prime}\right)$ and $=\phi_{1}\left(\bar{b}, \bar{a}_{2}^{\prime \prime}\right)$. Finally, $\bar{a}_{2}^{\prime \prime}$ witnesses that $\operatorname{tp}\left(\bar{a}_{1}^{\prime}\right)$ is consistent with $\phi_{1}(\bar{b}, \bar{x})$. $\mathbf{m}_{\text {Claim } 1}$

Find $\bar{c}=q$ such that $\operatorname{tp}\left(\bar{c} / \bar{a}_{2}\right)$ is isolated, which is possible by the remark after Lemma 2 . Let $\operatorname{tp}\left(\bar{c} / \bar{a}_{2}\right)$ be isolated by $\psi\left(\bar{z}, \bar{a}_{2}\right)$.

Claim 2. $\models \psi\left(\bar{c}^{\prime}, \bar{a}_{2}\right)$ implies $\bar{a}_{1} \downarrow \bar{c}^{\prime}$ and hence $\models \neg \phi_{1}\left(\bar{c}^{\prime}, \bar{a}_{1}\right)$.

Proof. Since $\bar{a}_{1} \downarrow \bar{a}_{2}, \operatorname{tp}\left(\bar{a}_{1} / \bar{a}_{2}\right)$ is strongly nonisolated, and $\operatorname{tp}\left(\bar{c}^{\prime} / \bar{a}_{2}\right)$ is isolated, we get $\bar{c}^{\prime} \downarrow \bar{a}_{1}\left(\bar{a}_{2}\right)$. By transitivity $\bar{c}^{\prime} \downarrow \bar{a}_{1} \bar{a}_{2}$ and $\bar{c}^{\prime} \downarrow \bar{a}_{1}$.

From Claim 2 we conclude that the following formula, call it $\theta(\bar{x})$, is satisfied by $\bar{a}_{1}$ (and hence belongs to $p$ ):

$$
\left(d_{p} \bar{z}\right)(\forall \bar{y})\left(\psi(\bar{y}, \bar{z}) \Rightarrow \neg \phi_{1}(\bar{y}, \bar{x})\right) .
$$

Thus $(\theta(\bar{x}) \wedge \varphi(\bar{x})) \in p$, and we can find $\bar{a}$ satisfying it such that $\operatorname{tp}(\bar{a})$ is isolated. Since, by Claim $1, \operatorname{tp}(\bar{a})$ is consistent with $\phi_{1}(\bar{b}, \bar{x})$ we assume without loss of generality that $=\phi_{1}(\bar{b}, \bar{a})$.

Pick $\bar{d}=p$ such that $\models \psi(\bar{b}, \bar{d})$. Since $p$ is stationary and strongly nonisolated and $\operatorname{tp}(\bar{a})$ is isolated we have $\bar{d} \downarrow \bar{a}$. Combining with $\models \theta(\bar{a})$ we derive $\models(\forall \bar{y})\left(\psi(\bar{y}, \bar{d}) \Rightarrow \neg \phi_{1}(\bar{y}, \bar{a})\right)$; using $\models \psi(\bar{b}, \bar{d})$ we get $\models \neg \phi_{1}(\bar{b}, \bar{a})$. A contradiction.

Proof of Theorem 1. Suppose that $p \in S(\emptyset)$ is a strongly nonisolated type. For each $M \models T$ and $r \in S(\emptyset)$ define $w_{r}(M)$ to be the largest possible integer $n$, if any, for which there exists $\bar{a}_{1} \ldots \bar{a}_{n}=r^{n}$ such that each $\bar{a}_{i}$ forks with $M$.

We shall construct models $M_{n}$ for $n \in \omega$ satisfying

$$
n \leq \lim _{k \rightarrow \infty} w_{p^{k}}\left(M_{n}\right)<2^{n},
$$

which clearly suffices to derive the desired conclusion.

Fix $n \in \omega$ and find $\bar{b}_{1}$ such that $\operatorname{tp}\left(\bar{b}_{1}\right)$ is $p^{k}$-minimal for almost all $k \in \omega$ : to see that this is possible, find $\bar{b}_{1}$ such that $\operatorname{tp}\left(\bar{b}_{1}\right)$ is $p^{k}$-minimal 
for some $k \geq 1$ and that $\operatorname{CB}\left(\operatorname{tp}\left(\bar{b}_{1}\right)\right)$ is minimal possible, say $\alpha$; then the formula isolating $\operatorname{tp}\left(\bar{b}_{1}\right)$ among the types of CB-rank at least $\alpha$ witnesses $p^{j}$-minimality of $\operatorname{tp}\left(\bar{b}_{1}\right)$ for all $j \geq k$. Further, find $\bar{b}_{2}$ such that $\operatorname{tp}\left(\bar{b}_{2} / \bar{b}_{1}\right)$ is $p^{k}$-minimal for almost all $k \in \omega, \ldots$, find $\bar{b}_{n}$ such that $\operatorname{tp}\left(\bar{b}_{n} / \bar{b}_{1} \bar{b}_{2} \ldots \bar{b}_{n-1}\right)$ is $p^{k}$-minimal for almost all $k \in \omega$. Therefore, for almost all $k \in \omega,\left(\bar{b}_{1}, \ldots, \bar{b}_{n}\right)$ is a $p^{k}$-minimal sequence. Let $M_{n}$ be prime over $\bar{b}_{1} \ldots \bar{b}_{n}$.

Fix $m \in \omega$ such that $\left(\bar{b}_{1}, \ldots, \bar{b}_{n}\right)$ is $p^{j}$-minimal for all $j \geq m$ and let $r=p^{m}$.

Claim 1. $n \leq w_{r}\left(M_{n}\right)$.

Proof. By induction, choose $\bar{a}_{1}, \ldots, \bar{a}_{n} \models r$ such that:

$$
\begin{array}{lr}
\bar{a}_{k} \downarrow \bar{b}_{1} \ldots \bar{b}_{k-1} & \text { for } k \geq 2, \\
\bar{a}_{k} \downarrow \bar{b}_{k}\left(\bar{b}_{1} \ldots \bar{b}_{k-1}\right) & \text { for } k \geq 1, \\
\bar{a}_{k} \downarrow \bar{a}_{1} \ldots \bar{a}_{k-1} \bar{b}_{1} \ldots \bar{b}_{n}\left(\bar{b}_{1} \ldots \bar{b}_{k}\right) & \text { for } k \geq 1 .
\end{array}
$$

The above choice is possible by $r$-minimality of $\left(\bar{b}_{1}, \ldots, \bar{b}_{n}\right)$.

We shall show that $\bar{a}_{k+1} \downarrow \bar{a}_{1} \ldots \bar{a}_{k} \bar{b}_{1} \ldots \bar{b}_{k}$ for $1 \leq k \leq n-1$.

For $1 \leq j \leq k \leq n$, from $(3)_{j}$ we have

$$
\bar{a}_{j} \downarrow \bar{a}_{1} \ldots \bar{a}_{j-1} \bar{b}_{k+1}\left(\bar{b}_{1} \ldots \bar{b}_{k}\right),
$$

which, for $j=1, \ldots, k$, implies that $\left\{\bar{a}_{1}, \ldots, \bar{a}_{k}, \bar{b}_{k+1}\right\}$ is an independent set over $\bar{b}_{1} \ldots \bar{b}_{k}$. Thus

$$
\bar{b}_{k+1} \downarrow \bar{a}_{1} \ldots \bar{a}_{k}\left(\bar{b}_{1} \ldots \bar{b}_{k}\right) .
$$

From $(3)_{k+1}$ we have $\bar{a}_{k+1} \downarrow \bar{a}_{1} \ldots \bar{a}_{k}\left(\bar{b}_{1} \ldots \bar{b}_{k} \bar{b}_{k+1}\right)$. By transitivity, from the last two independence relations, we get

$$
\bar{a}_{k+1} \bar{b}_{k+1} \downarrow \bar{a}_{1} \ldots \bar{a}_{k}\left(\bar{b}_{1} \ldots \bar{b}_{k}\right)
$$

and hence

$$
\bar{a}_{k+1} \downarrow \bar{a}_{1} \ldots \bar{a}_{k}\left(\bar{b}_{1} \ldots \bar{b}_{k}\right),
$$

which combined with $(1)_{k+1}$ implies

$$
\bar{a}_{k+1} \downarrow \bar{a}_{1} \ldots \bar{a}_{k} \bar{b}_{1} \ldots \bar{b}_{k} .
$$

Therefore, $\bar{a}_{1} \ldots \bar{a}_{n} \models r^{n}$. By $(2)_{k}$ each $\bar{a}_{k}$ forks with $\bar{b}_{1} \ldots \bar{b}_{n}$ so we conclude that $n \leq w_{r}\left(M_{n}\right)$. $\mathbf{m}_{\text {Claim } 1}$

Claim 2. $w_{r}\left(M_{n}\right)<2^{n}$.

Proof. Suppose that $\bar{a}_{1} \ldots \bar{a}_{2^{n}}=r^{2^{n}}$ and split it into two disjoint sequences of length $2^{n-1}$ each. By our choice of $k, \operatorname{tp}\left(\bar{b}_{1}\right)$ is $r^{2^{n-1}}$-minimal so, by Lemma $3, \bar{b}_{1}$ is independent of at least one of our sequences, say $\bar{a}_{1} \ldots \bar{a}_{2^{n-1}}$. Further, $\operatorname{tp}\left(\bar{b}_{2} / \bar{b}_{1}\right)$ is, by our assumption, $r^{2^{n-2}}$-minimal, so after splitting $\bar{a}_{1} \ldots \bar{a}_{2^{n-1}}$ into two disjoint sequences of equal length we conclude using Lemma 3 that, without loss of generality, $\bar{b}_{2} \downarrow \bar{a}_{1} \ldots \bar{a}_{2^{n-2}}\left(\bar{b}_{1}\right)$. 
Combining with the previous we get $\bar{b}_{2} \bar{b}_{1} \downarrow \bar{a}_{1} \ldots \bar{a}_{2^{n-2}}$. Continuing in this way, we end up with, say, $\bar{a}_{1}$ independent of $\bar{b}_{1} \ldots \bar{b}_{n}$. Since $r$ is strongly nonisolated and since $M_{n}$ is atomic over $\bar{b}_{1} \ldots \bar{b}_{n}$ we conclude that $\bar{a}_{1}$ is independent of $M_{n}$. Therefore $w_{r}\left(M_{n}\right)<2^{n}$. Claim 2

Note that for any type $q$ the sequence $\left(w_{q^{i}}(M) \mid i \in \omega\right)$ is nondecreasing, so that $\lim _{k \rightarrow \infty} w_{p^{k}}\left(M_{n}\right)$ exists. From Claims 1 and 2 we have

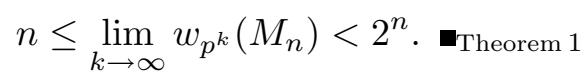

\section{References}

[1] J. Baldwin, Fundamentals of Stability Theory, Springer, 1988.

[2] E. Hrushovski, Finitely based theories, J. Symbolic Logic 54 (1989), 221-225.

[3] B. Herwig, J. Loveys, A. Pillay, P. Tanović and F. O. Wagner, Stable theories without dense forking chains, Arch. Math. Logic 31 (1992), 297-303.

[4] A. Lachlan, On the number of countable models of a countable superstable theory, in: Logic, Methodology and Philosophy of Sciences, P. Suppes et al. (eds.), NorthHolland, 1973, 45-56.

[5] M. Makkai, A survey of basic stability theory with particular emphasis on orthogonality and regular types, Israel J. Math. 49 (1984), 181-238.

[6] A. Pillay, Countable models of stable theories, Proc. Amer. Math. Soc. 89 (1983), 666-672.

[7] A. Tsuboi, On theories having a finite number of nonisomorphic countable models, J. Symbolic Logic 50 (1985), 806-808.

[8] -, Countable models and unions of theories, J. Math. Soc. Japan 38 (1986), 501-508.

Matematički institut SANU

Knez Mihajlova 35

11001 Beograd, Serbia, Yugoslavia

E-mail: tane@mi.sanu.ac.yu

Received 12 April 2000;

in revised form 5 February 2001 